\title{
Research of reliability of urban electric transport system
}

\author{
$A E$ Auhadeev ${ }^{1, *}, R S$ Litvinenko $^{1}, V P$ Fandeev $^{2}, P P$ Pavlov $^{1}, V M$ Butakov $^{1}$ and $A R$ Litvinenko $^{3}$ \\ ${ }^{1}$ Federal State Budgetary Institution of Higher Education "Kazan State Power Engineering University", Kazan, Krasnoselskaya Street, \\ 51, 420066, Russian Federation \\ ${ }^{2}$ Federal State Budgetary Institution of Higher Education "Penza State University", Penza, Krasnaya Street, 40, 440026, Russian \\ Federation \\ ${ }^{3}$ Federal State Autonomous Educational Institution of Higher Education "St. Petersburg State University of Aerospace Instrumentation", \\ St. Petersburg, Bolshaya Morskaya Street, 67A, 190000, Russian Federation
}

\begin{abstract}
Modern methods for analyzing and assessing the reliability of complex technical systems consider only its individual elements. The specifics of structural-functional interactions and integration into subsystems of different hierarchical levels are not taken into account. Studies show that analyzing the reliability of urban electric transport, also focus on individual components: the reliability of electric traction rolling stock, the reliability of the power supply system, the reliability of the track infrastructure, etc. The authors propose an approach that allows to consider urban electric transport as a complex technical system consisting of several levels that are hierarchically subordinate. Each level combines the components directly involved in the transmission and transformation of electric power into mechanical operation of the rolling stock under the influence of the external environment by means of traction electrical equipment. This approach will allow to develop the theoretical foundations of methods for analyzing the impact of the reliability of individual components of a complex technical system of urban electric transport on the effectiveness of the electric traction implementation.
\end{abstract}

\section{Introduction}

In the system of urban passenger traffic, such types of electric transport as the trolleybus, tram and metro are mainly used due to their high productivity and environmental friendliness. The main feature of such transport is the use of electric traction, which provides transmission and conversion of electric power by traction electrical equipment (TEE) into mechanical work of rolling stock through the provision of technological and production tasks of the transport process $[1,2]$.

Under the urban electric transport (UET) system, in terms of its technical implementation, we will understand the production functioning and developing system, which is a combination of electric vehicles, transport infrastructure, transport enterprises and management systems aimed at the most complete satisfaction of the needs of the urban population on trips within the urban agglomeration.

UET can be considered as a complex technical system (CTS) [1, 3]. In accordance with [4], by the system we mean a set of elements defined in time and space with known properties and with ordered connections between the elements and properties oriented towards the fulfillment of the main task of the given set. In some cases [5] use the term complex dynamic system - the formation of elements of different nature, which has functions and properties that are missing from each of the elements and is able to function, statically correlating in a certain range with the environment and, due to this, maintain its structure during continuous changes in the interactions of elements according to complex dynamic laws. UET also exists among other objects, united by the concept of "external environment". The objects of the external environment are connected with the elements of the system; however, this connection is significantly weaker than the internal connections in the system. Elements are parts or components of a system conditionally accepted as indivisible. The internal structure and properties of an element are not the subject of research and study, only such properties are significant, which manifest themselves in the interaction with other elements and affect the properties of the system as a whole. The division of the system into elements is relative and depends on the task and objectives of the study; otherwise, in other aspects, the element can be considered as a CTS. The combination (integrity) of elements and properties is an important distinctive criterion of the system.

Also for CTS is peculiar the possibility of dividing it into subsystems - a set of system elements that function independently [5]. In this case, the goals and objectives of the functioning of the subsystem itself, possessing some separateness, are subordinated to the general goals of the system.

\footnotetext{
* Corresponding author: auhadeevkgma@yandex.ru
} 


\section{Materials of theoretical study}

Full and effective use of UET for the implementation of the passenger transportation process largely depends on the technical condition of its elements and is largely determined by the reliability of their operation. In accordance with [6], reliability is understood as the property of readiness and reliability and repairability properties affecting it, and maintenance support. Previously, the definition of this term [7] was the following: reliability is the property of an object to preserve, within the established limits, the values of all parameters characterizing the ability to perform the required functions in specified modes and conditions of use, maintenance, storage and transportation. Reliability is a complex property, which, depending on the purpose of the object and the conditions of its use, may include reliability, durability, maintainability and persistence, or certain combinations of these properties. The effectiveness of its application largely depends on the reliability of the equipment - the ability to meet the requirements for the service with given quantitative characteristics [6], which depends on a combination of capabilities and readiness for use. Therefore, UET should be considered as a single CTS, consisting of a large number of different elements that interact to ensure an efficient electric traction process.

Analysis of scientific literature allows us to conclude that the consideration of the issues of reliability of UET is mainly focused on the assessment of the reliability of its constituent elements and does not imply their synthesis into a single CTS. Failures of individual elements do not lead to the loss of efficiency and efficiency of the entire UET, but the pace and quality of the performance of the specified functions is reduced, so an objective need arises in assessing the reliability of the entire system as a whole. Highlight the following main areas of research on the operational reliability of UET elements can be distinguished: reliability of electric rolling stock [8-11]; reliability of the traction power supply system $[12,13]$; reliability of the track route [1416].

There is a large amount of literature devoted to the reliability of individual units, assembly units and assemblies of different physical nature and purpose, which are used both as separate elements and as part of technical devices. Evaluating the reliability of UET, a systematic approach is needed, which involves first calculating the reliability indices of simpler subsystems and elements, and then grouping the results obtained in a certain way in order to obtain the characteristics of the system.

In the structure UET allocate the following technical means $[1,3]$ : electric rolling stock (ERS); track facilities and roads that form the transport network; plants and depots providing maintenance and repair of ERS; power supply devices; communication and blocking devices; means of organizing and controlling traffic. Figure 1 shows the interaction between these subsystems.

The reliability study of UET, as a CTS consisting of a large number of elements, is proposed to use the following subsystems directly involved in the transmission and transformation by means of a feasibility study of electric power into the mechanical operation of rolling stock under environmental conditions: traction ERS subsystem; path subsystem; traction power supply subsystem.

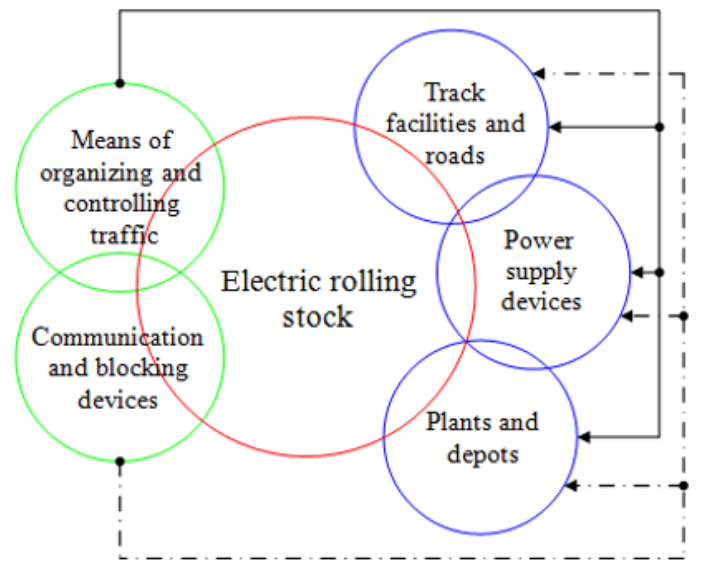

Fig. 1. Diagram of the interaction of subsystems CTS.

Further specification and formation of additional subsystems in the composition of the UET is not always justified in the analysis of reliability, because:

- the required level of reliability of the equipment is laid at the development stage, and then, through proper operation and timely maintenance, it can only be maintained. It is possible to increase the achieved level of reliability only by upgrading the technical object itself. In this regard, it is possible to omit consideration of the issues of reliability of the means of organizing and controlling traffic, since the issues of efficient use of ETS, in this context, belong to the field of theories of electric traction and transport logistics;

- in spite of the fact that ERS is a renewable CTS, the reliability of plants and depots providing maintenance and repair of ERS does not affect the reliability of the system as a whole, but rather depends on the professionalism and training of personnel [17]. Therefore, in the formation of mathematical models, it is possible to accept the assumption that the restoration of the failed elements is carried out within the deadlines set by the regulatory and technical documentation;

- the mathematical model of any CTS should not be "overloaded" by taking into account all sorts of factors, as this leads to complication of calculations, and often to distortion of the results and the inadequacy of the model to the original. Therefore, when forming the structure and composition of ETSG, it is necessary to be guided by the necessary completeness and reliability of a certain part of the properties (in our case, reliability) of the original, which is essential precisely in this study and in the given formulation of the problem.

According to the system approach to assess the impact of reliability on the efficiency and quality of the operation of UET, it is advisable to apply a structural decomposition method, the essence of which is to divide the system under study into subsystems (components) of a lower hierarchy level, which will replace the solution of one large task with a solution of a series of tasks of 


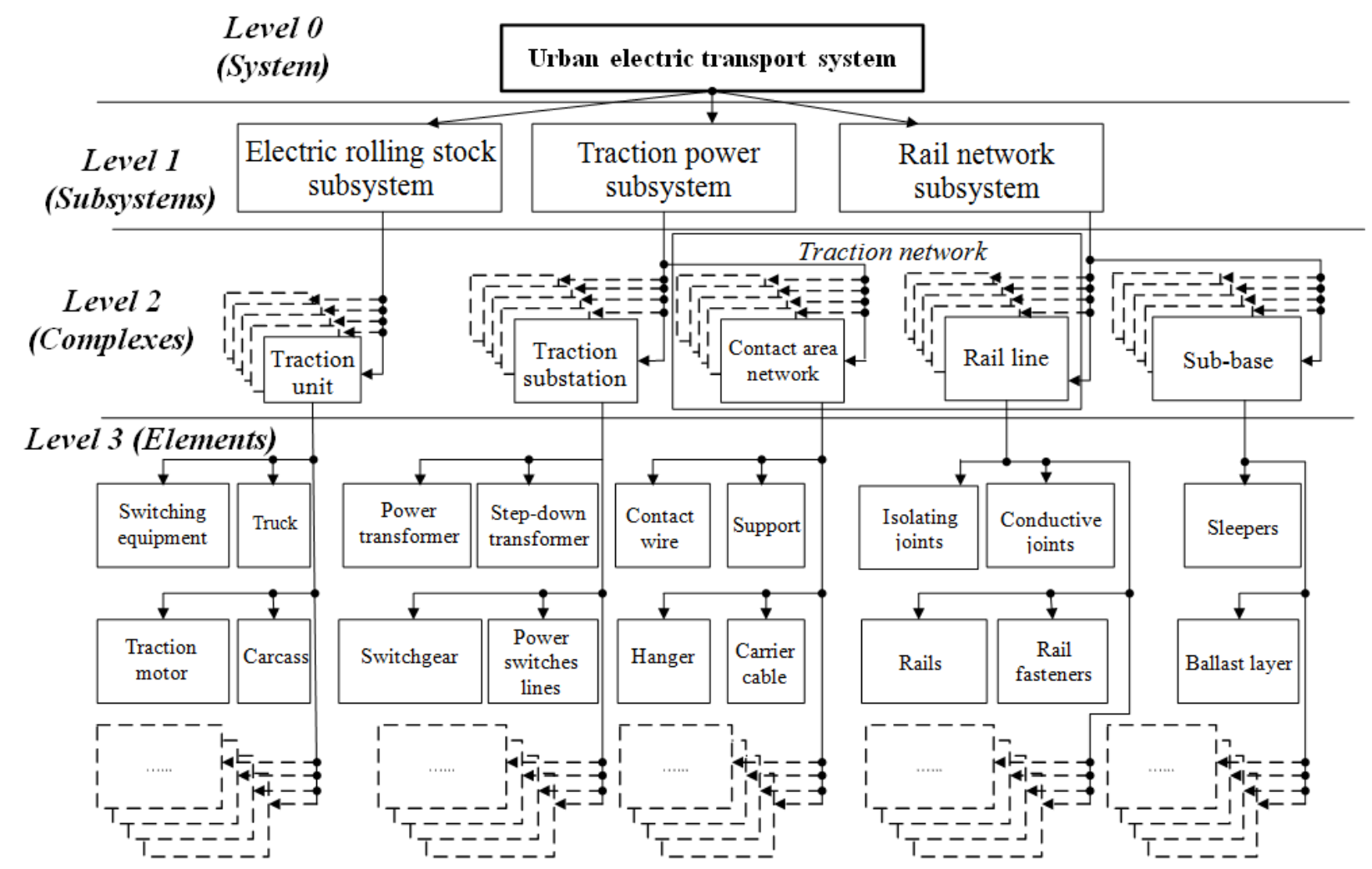

Fig. 2. Decomposition of the electric transport system of the urban agglomeration.

less complexity. Figure 2 presents a variant of the UET decomposition for the purpose of the subsequent analysis of its reliability, assuming the selection of four levels in the system structure.

UET is located at the initial level; subsystems of electric rolling stock, traction power supply and rail network are located at the first level (in the case of use as a ERS, tram or subway cars); the second level is represented by electrical and engineering systems that are part of the subsystems of the 1st level; on the third (lower) level are the elements of the complexes (TEE and mechanical equipment). It is also possible to select components of a lower level in the UET elements assembly units, assemblies, units; however, as mentioned above, the reliability assessment of these technical means (devices) has been studied and studied sufficiently earlier. Therefore, such a degree of detail of the description and the number of levels of the hierarchical structure of UET, such as the CTS, can be considered sufficient and convenient for perception, and also corresponds to the modern level of knowledge of a specialist in the field of reliability [17].

\section{Conclusion}

The quality of the operation of UET is estimated by the performance indicator, hence the task of assessing the reliability of the system is reduced to determining its effect on the system's efficiency. For these purposes, it is convenient to use a complex indicator - the coefficient of efficiency preservation. In accordance with [18], the coefficient of efficiency $\mathrm{K}$ ef retention is the ratio of the value of the indicator of the effectiveness of a product for a certain period of operation $\mathrm{W}(\mathrm{t})$ to the nominal value of this indicator W 0 (t) calculated under the condition that no product fails during this period. Calculated in such a way K_ef, it will be possible to estimate the deviation of the calculated parameters of the efficiency of the UET functioning from the nominal value, taking into account the reliability of its constituent elements, and, if necessary, take measures aimed at raising it.

Thus, the task of analyzing ETSC as an object of research into reliability comes down to assessing the reliability of its subsystems, complexes and elements, and the subsequent synthesis of the results obtained. This approach is possible both at the stage of creating a new UET in a urban agglomeration, and in relation to existing megacities in the process of modernizing transport routes by introducing new sections.

\section{References}

1. N.I. Shchurov, Methods and means of saving and increasing energy efficiency in the system of urban electric transport, 385 (2003)

2. L.N. Kisneeva, A.E. Aukhadeev, D.I. Tukhbatullina, U.V. Staroverova, Description of urban electric trance as a complex technical system, Modern Science, 11, 181 - 183 (2017)

3. Yu.A. Rylov, A.E. Aukhadeev, N.Yu. Egorova, P.S. Urzhumtsev, Electric traction in urban electric 
transport system, Modern Science, 11, 195-197 (2017)

4. A.I. Gaykovich, Basics of the theory of designing complex technical systems, 432 (2001)

5. V.I. Sopov, N.I. Shchurov, Modeling of electric transport systems, 189 (2005)

6. GOST R 27.002 - 2009. Reliability in engineering. Terms and definitions (Moscow: Standardinform) $p$ 27

7. GOST 27.002-89. Reliability in technology. Terms and Definitions. (Moscow: Publishing Standards) p 37

8. A.V. Prokhorov, Reliability of the electrical insulation system of traction motors, Energy Security and Energy Saving, 2, 21-25 (2010)

9. N.S. Bachurin, Methods for assessing the reliability indicators of a tram car, Ural Transport, 1(20), 36 39 (2009)

10. I.Yu. Mikhailov, O.P. Muravlev, A.L. Fedyanin, Engineering analysis of the operational reliability of trolleybus electrical equipment, Bulletin of Kuzbass State Technical University, 3(115), 85-92 (2016)

11. A.M. Hudonogov, D.A. Olentsevich, E.M. Lytkina, V.N. Ivanov, Reliability of asynchronous auxiliary machines for electric locomotives, Bulletin of Irkutsk State Technical University, 4(36), 117-119 (2008)

12. P-639. Recommendations on the main provisions of the study of the reliability of the contact network devices. Organization of Cooperation of Railways (Warsaw), 16 (2005)

13. V.A. Anischenko, I.V. Kolosova, Reliability of power supply systems, 151 (2007)

14. V.S. Lysyuk, V.T. Semenov, Robust and reliable railway, 589 (2003)

15. V.S. Lysyuk, V.B. Kamensky, Reliability of the railway track, 286 (2001)

16. G.K. Shchepotin, Operational reliability of a railway track, 144 (2008)

17. E.M. Khusnutdinova, G.R. Khamidullina, P.P. Pavlov, A.E. Aukhadeyev, R.S. Litvinenko, A.N. Khusnutdinov, Training transport specialists based on a contemporary view of self-organization of sophisticated developing systems, IOP Conference Series: Materials Science and Engineering, 412(1), 012047 (2018)

18. GOST R 53480 - 2009 Reliability in engineering. Terms and Definitions. (Moscow: Standardinform) p 33 